\title{
The influence of ant-attendance on aphid behaviour investigated with the electrical penetration graph technique
}

\author{
Gisep Rauch ${ }^{1,3}$, Jean-Christophe Simon ${ }^{2}$, Bernard Chaubet ${ }^{2}$, Lucie Haack ${ }^{2}$, Thomas Flatt ${ }^{1,4}$ \& \\ Wolfgang W. Weisser ${ }^{1,3, *}$ \\ ${ }^{1}$ Zoology Institute, University of Basel, Rheinsprung 9, 4051 Basel, Switzerland; ${ }^{2}$ INRA/ENSAR UMR 'Biologie \\ des Organismes et des Populations appliquée à la Protection des Plantes' (BiO3P), INRA B.P. 29, 35653 Le Rheu \\ Cedex, France; ${ }^{3}$ Present address: Institute of Ecology, Friedrich-Schiller-University, Dornburger Str. 159, 07743 \\ Jena, Germany; ${ }^{4}$ Present address: Department of Biology, University of Fribourg, Ecology and Evolution Unit, \\ Chemin du Musée 10, 1700, Fribourg, Switzerland; *Author for correspondence
}

Accepted: November 13, 2001

Key words: EPG, ants, aphids, mutualism, behaviour, Metopeurum fuscoviride, Lasius niger

\begin{abstract}
For the mutualistic interaction between the aphid Metopeurum fuscoviride Stroyan (Homoptera: Aphididae) and the ant Lasius niger L. (Hymenoptera: Formicidae) it has been shown that ant-tended aphids develop faster, reproduce at a higher rate, and live longer than aphids not tended by ants. We used electrical penetration graphs (EPG) to investigate if behavioural patterns differ between ant-tended and untended $M$. fuscoviride during $8 \mathrm{~h}$ experiments. Measurements were made on adult aphids from four different ant-tended colonies that continued to be tended by L. niger during the experiments, and from four different colonies where ant workers were excluded several days before the start of the experiment and that were also not tended by ants during the experiments. Ants readily tended wired aphids and ant tending did not interfere with the EPG measurements. There were no significant differences in the duration of sieve element penetration or in any other analysed feeding-related EPG parameters between anttended and untended individuals. However, the quality of the EPG recordings did not allow the distinction between the EPG-waveform E1 (salivation only) and E2 (salivation and ingestion). These results suggest that the changes in life-history traits of ant-tended aphids do not result from changes in time of sieve element penetration waveforms. Alternative mechanisms may involve an increase in the rate of sap uptake or a higher effectiveness in nutrient uptake in the presence of ants. Our study demonstrates that the EPG technique is a useful tool to investigate the feeding behaviour of aphids during interactions with ants.
\end{abstract}

\section{Introduction}

Among aphids (Homoptera: Aphididae), many species display mutualistic interactions with ants (Herzig, 1937; Nixon, 1951; Buckley, 1987). For ants, the main benefit is the provision of carbohydrates by the aphids (Buckley, 1987). One of the main benefits for aphids is protection against natural enemies (Buckley, 1987). However, aphids may also benefit from ant tending when natural enemies are absent. A number of studies have shown that survival and fecundity of aphids are positively affected by ant tending (Banks, 1958; Buckley, 1987). For example, in experiments with the ant-tended aphid Metopeurum fuscoviride Stroyan, single ant-tended individuals developed faster, had a higher rate of reproduction and lived longer than individuals not tended by ants (Flatt \& Weisser, 2000).

While the positive effects of ant tending on aphid performance have been shown repeatedly, the mechanisms underlying these effects are still unclear. For example, Herzig (1937) showed that ant-tended aphids have an increased honeydew production, by counting the number of excreted honeydew droplets of anttended and untended aphids and calculating the total volume of honeydew produced per day. This increased 
honeydew production has been hypothesised to result in a faster rate of nutrient assimilation (Herzig, 1937; Banks \& Nixon, 1958; Buckley, 1987). However, the increased honeydew production in tended aphids could be due to a prolonged time spent in the sieve elements, to an increase of sap uptake per time unit spent in the sieve elements, or a combination of both. To distinguish between these different mechanisms, the behaviour of ant-tended and untended individuals must be analysed. A number of studies have shown that aphid behaviour can reveal some insight into the mechanisms underlying changes in aphid performance. For example, Chongrattanameteekul et al. (1991a, b) investigated the effects of aphid density on behaviour and performance in Rhopalosiphum padi L. Aphids at low density displayed a reduced time of nonprobing and an increased time of probing plus food ingestion as compared with individuals at higher density. Longevity and the number of offspring produced were increased in the low density treatment, suggesting that the change in behaviour was the cause of the difference in performance. Consistent with this hypothesis, the same study on another aphid (Sitobion avenae $\mathrm{F}$.) found that density had no effect on feeding behaviour and also no differences in aphid longevity and fecundity could be detected.

A well-established experimental approach to analyse the behaviour of aphids is the use of electrical penetration graphs (EPG; McLean \& Kinsey, 1964; Tjallingii, 1986, 1990). The EPG technique has been successfully employed to compare aphid behaviour on resistant and susceptible plants (Spiller, 1988; Caillaud et al., 1995), to study aphid behaviour in relation with virus transmission (Prado, 1997), or to investigate the process of host plant acceptance (Tjallingii \& Mayoral, 1992). In this study we use EPG to investigate whether ant tending influences the behaviour of the aphid $M$. fuscoviride in a way that could explain the increase in aphid performance in the presence of ant workers. We hypothesised that ant-tended individuals spend more time in the sieve elements than untended aphids.

\section{Materials and methods}

Organisms. Metopeurum fuscoviride is a monophagous, holocyclic aphid species that lives on common tansy Tanacetum vulgare L. (Asteraceae). Metopeurum fuscoviride is regularly tended by the ant Lasius niger L. Two ant colonies were collected at a field site near Basel, Switzerland in June 1999, and kept in plastic boxes $(45 \times 34 \times 30 \mathrm{~cm})$ coated with fluon (Northern Products Inc., Woonsocket, Rhode Island, USA) and talcum powder to prevent escape. All boxes were filled with natural substrate. The ant colonies had access to $M$. fuscoviride aphids through plastic tubing $(\varnothing 1 \mathrm{~cm})$. Ants were regularly fed with freshly-killed fruit flies (Drosophila melanogaster Meigen) and pea aphids (Acyrthosiphon pisum Harris), and sprayed with tap water to avoid desiccation.

In 1996, aphids and tansy plants were collected at the same field site near Basel, and an aphid population was established on potted plants in the garden of the Zoology Institute Basel. Each autumn, aphids produced sexual individuals and populations overwintered as diapausing eggs. For the experiment, parthenogenetic lines stemming from sexual reproduction were randomly selected from the population in spring 1999 and used to establish aphid colonies. To obtain experimental plants, ramets of the host plant T. vulgare were planted singly in plastic pots $(\varnothing$ $10 \mathrm{~cm}$, height $7.4 \mathrm{~cm}$ ) filled with commercial growing medium (TKS ${ }^{\circledR}$ 2, Floragard VertriebsGmbH, D26129 Oldenburg, Germany) in spring 1999. The experimental plants were $20-40 \mathrm{~cm}$ high when used in the experiments.

All material was transported to the Institut $\mathrm{Na}$ tional de la Recherche Agronomique (INRA) in Rennes, France, where the experiments took place in July 1999. All plants and animals were kept in climatised rooms at $20 \pm 1{ }^{\circ} \mathrm{C}$ and a photoperiod of L16:D8.

Experimental setup. Two experimental treatments were established. In the ant-tended treatment, individuals were taken from aphid colonies that were ant-tended for several generations. In the untended experiment, aphid colonies were separated from ant colonies at least six and maximally 18 days before the measurements were made. Only adult individuals were used to prevent problems due to moulting of larvae during EPG-recording. In total, individuals from eight different aphid colonies on eight different tansy plants were used in the experiment (four colonies in the ant-tended and four colonies in the untended treatment).

An experimental aphid was chosen randomly from the colony half an hour before the start of the experiment. For manipulation, the aphid was placed on the opening of a plastic tube connected to a vacuum pump. A $25 \mu \mathrm{m}$ gold wire was then attached to the dorsum of 
the aphid using conductive silver paint. After the wire was securely fixed, the individual was placed singly on an experimental plant and connected to EPG measurement instrument (Tjallingii, 1990, see below for details on EPG).

Behaviour of four aphids on four experimental plants were analysed simultaneously, two aphids from different ant-tended aphid colonies and two aphids from different untended aphid colonies. Aphids from ant-tended colonies were also exposed to ant attendance during EPG recording, whereas those from untended colonies continued to be deprived of ants during the experiments. The two plants for the anttended treatment were placed in a box $(45 \times 34 \times$ $10.5 \mathrm{~cm}$ ) coated with fluon and talcum powder and connected with a plastic tube to the ant colony. To reduce the time period until ants discovered the single aphid on the experimental plant, an aphid colony was placed in the box during the night preceding an experiment and removed in the morning. When the two experimental plants were placed in the box in the morning, ant workers almost immediately searched these plants and started tending the aphids. The two plants for the untended treatment were also placed in a box $(45 \times 34 \times 10.5 \mathrm{~cm})$ coated with fluon and talcum powder. The two boxes were put in a Faraday cage where the EPG recordings were made. The relative positions of the plants for the untended and ant-tended treatment were changed daily. All experimental plants were used only once.

Experiments started at 10 a.m. and lasted for $8 \mathrm{~h}$. Aphids that fell off the plant were immediately put back with a fine brush. Therefore, the interruption of feeding, caused by falling off the plant of an aphid, should not be substantially different from the interruption of feeding of an aphid walking. Only data of individuals showing stylet penetration into the plant were used for analysis. In total, measurements were made on four aphid colonies $\times$ three individuals $=12$ replicates in the ant-tended treatment with the first ant colony. The same four aphid colonies were used for measurements with the second ant colony, which gave in total 24 replicates in the ant-tended treatment. In the untended treatment we measured four aphid colonies $\times$ four individuals $=16$ replicates. Temperatures during the whole experimental period (10 days) ranged between $24^{\circ} \mathrm{C}$ and $29^{\circ} \mathrm{C}$. Mean temperature change during the $8 \mathrm{~h}$ recording sessions was $1.6^{\circ} \mathrm{C} \pm 0.22^{\circ} \mathrm{C}$ $(N=10$ days $)$.
Electronic recording. The output of each of the four electrical penetration graph (EPG) amplifiers ( $10^{9} \mathrm{Ohm}$ impedance DC amplifier) was connected to an analogue-to-digital converter (Metrabyte Dash/8). Digitalized data were analysed with the software 'MacStylet' (Febvay et al., 1996).

Statistical analysis. Averages are presented as means \pm s.e. throughout the text. Data were tested for significance $(\mathrm{P}<0.05)$ using SAS version 6.12 (SAS Institute, 1989). Before we tested for differences between the ant-tended and untended treatment using $t$-tests, we tested whether there were differences between the different ant colonies and aphid colonies used in the experiment, separately for the tended and untended treatment. Where necessary, data were logtransformed to homogenise variances and/or restore normality.

\section{Results}

Behaviour of ants and EPG-measurements. Aphids were tended almost immediately after the start of the experiment. Tending levels in the experiment were high: at any time, at least one ant worker was present near the aphid, and frequently several workers were tending the wired individual. Wiring of aphids caused no obvious change in ant behaviour and no workers were observed attacking or biting into the wire. When ants were touching the wire, the signal showed strong oscillations for one or two seconds, but the different waveforms were still recognisable. Because the beginning and the end of the waveforms were marked manually on the output, these disturbances in the signals had no influence on the analysis.

Variables analysed. Table 1 lists the variables analysed in this study. These variables correspond to waveforms commonly recognised in studies of aphid behaviour (e.g., Tjallingii, 1990; Febvay et al., 1996). Total time of penetration in the sieve elements was of central interest for our study and its waveform $E_{\text {total }}$ was well recognisable. We were not able to distinguish between the $\mathrm{E} 1$ waveform at the intracellular voltage level (sieve element penetration followed by salivation) and the E2 waveform (sieve element ingestion and concurrent salivation). This was probably due to a high level of noise within the E waveform. $E_{\text {total }}$ can be assumed to be associated with the E2 waveform, provided that $E_{\text {total }}$ is on intracellular voltage level and 
Table 1. Behavioural parameters quantified from EPG-recordings over $8 \mathrm{~h}$ periods. See text for explanations

\begin{tabular}{|c|c|}
\hline Variable & Definition \\
\hline NP & Total time spent without any penetration (non-penetration time) \\
\hline$E_{\text {total }}$ & Total time of penetration in the sieve elements $(\mathrm{E} 1+\mathrm{E} 2)$ \\
\hline$E_{>8 \min }$ & $\begin{array}{l}\text { Total time of sieve element penetrations longer than } 8 \mathrm{~min} \text { (with subtraction of } \\
\text { the first } 8 \mathrm{~min} \text { of each penetration) }\end{array}$ \\
\hline$E_{\text {mean }}$ & Average time in sieve element per sieve element penetration longer than $8 \mathrm{~min}$ \\
\hline 1 st $E_{>8 \min }$ & Time to first sieve element penetration longer than $8 \mathrm{~min}$ \\
\hline $\mathrm{nE}$ & Number of sieve element penetrations longer than $8 \mathrm{~min}$ \\
\hline$E_{\text {long }}$ & $\begin{array}{l}\text { Duration of the longest single interval of continuous sieve element penetration } \\
\text { longer than } 8 \text { min during the experiment }\end{array}$ \\
\hline$E_{\text {last }}$ & $\begin{array}{l}\text { Duration of the last interval of continuous sieve element penetration longer than } \\
8 \text { min before the end of the experiment }\end{array}$ \\
\hline$P_{\text {rest }}$ & Total time of pathway and xylem phases and short potential drops \\
\hline
\end{tabular}

is sustained for more than $8 \mathrm{~min}$ (Tjallingii, 1990). We calculated $E_{>8 \min }$ as the total time of sieve element penetrations that lasted longer than $8 \mathrm{~min}$, by subtracting the first $8 \mathrm{~min}$ of each sieve element penetration. All waveforms of pathway and xylem phases (i.e., A, B, C, E1 at extracellular voltage level, F, and G) and stylet tip punctures of cell membranes (short potential drops, pd; cf., Tjallingii, 1990) were totalled as the variable $P_{\text {rest }}$ (Table 1).

Effects of ant and aphid colonies. Among the anttended replicates, ant colony identity did not affect any of the variables measured ( $t$-tests; $\mathrm{P}>0.05)$. We therefore pooled the data from both ant colonies together. Similarly, there were no significant differences between the four aphid colonies in any of the variables measured, when the ant-tended treatment and the untended treatment were analysed separately (ANOVA; $P>0.05$ ). We therefore pooled the data from the four aphid colonies together, both in the ant-tended treatment and in the untended treatment.

Effects of ant-attendance on aphid behaviour. When analysed over the entire $8 \mathrm{~h}$ measurement period, behaviour did not differ between the ant-tended and untended aphids in any of the variables analysed ( $t$ tests; $\mathrm{P}>0.05$; Figure 1 ). During $8 \mathrm{~h}$, ant-tended aphids penetrated the sieve elements $1.33 \pm 0.34$ times, untended aphids $1.69 \pm 0.33$ times. Anttended individuals penetrated sieve elements for $16 \%$ of their time while untended aphids spent $20 \%$ in the sieve-elements, but the difference was not significant (Figure 1).

To test whether the aphids needed some time to settle down on the tansy plants, behaviour in the first $4 \mathrm{~h}$ of measurement was compared to the behaviour in the second $4 \mathrm{~h}$ period, separately for each treatment. First, we again tested for significant ant colony and aphid colony effects, separately for each treatment and period. Of all tested variables, only NP in the first $4 \mathrm{~h}$ period was affected by the variable ant colony $(t=3.64$, d.f. $=22, \mathrm{P}=0.002)$ and was therefore not used for further analyses. Aphid behavioural parameters did not differ between different aphid colonies, except for $E_{\text {mean }}$ in the first four hours in the untended treatment $\left(F_{3,3}=9.44, P=0.049\right)$. This variable was therefore also not used for further analyses. All the other variables did not show a significant effect of the ant and aphid colonies and data from the different ant and aphid colonies were therefore pooled together.

Between the first and the second $4 \mathrm{~h}$ period, there were differences in only two of the variables analysed. In the ant-tended treatment, $E_{\text {total }}$ and $E_{>8 \text { min }}$ were significantly lower in the first period than in the second period ( $E_{\text {total }}: t=3.01$, d.f. $=22, \mathrm{P}=0.006$; $E_{>8 \min }: t=2.64$, d.f. $=22, \mathrm{P}=0.015$ ). No significant differences were found in $E_{\text {mean }}$ and Prest $(t$-tests; $\mathrm{P}>0.05)$. In the untended treatment, behavioural waveforms in the first $4 \mathrm{~h}$ period did not differ from the behavioural waveforms in the second four hour period in any of the variables analysed ( $t$-tests; $\mathrm{P}>0.05$; Figure 2). 


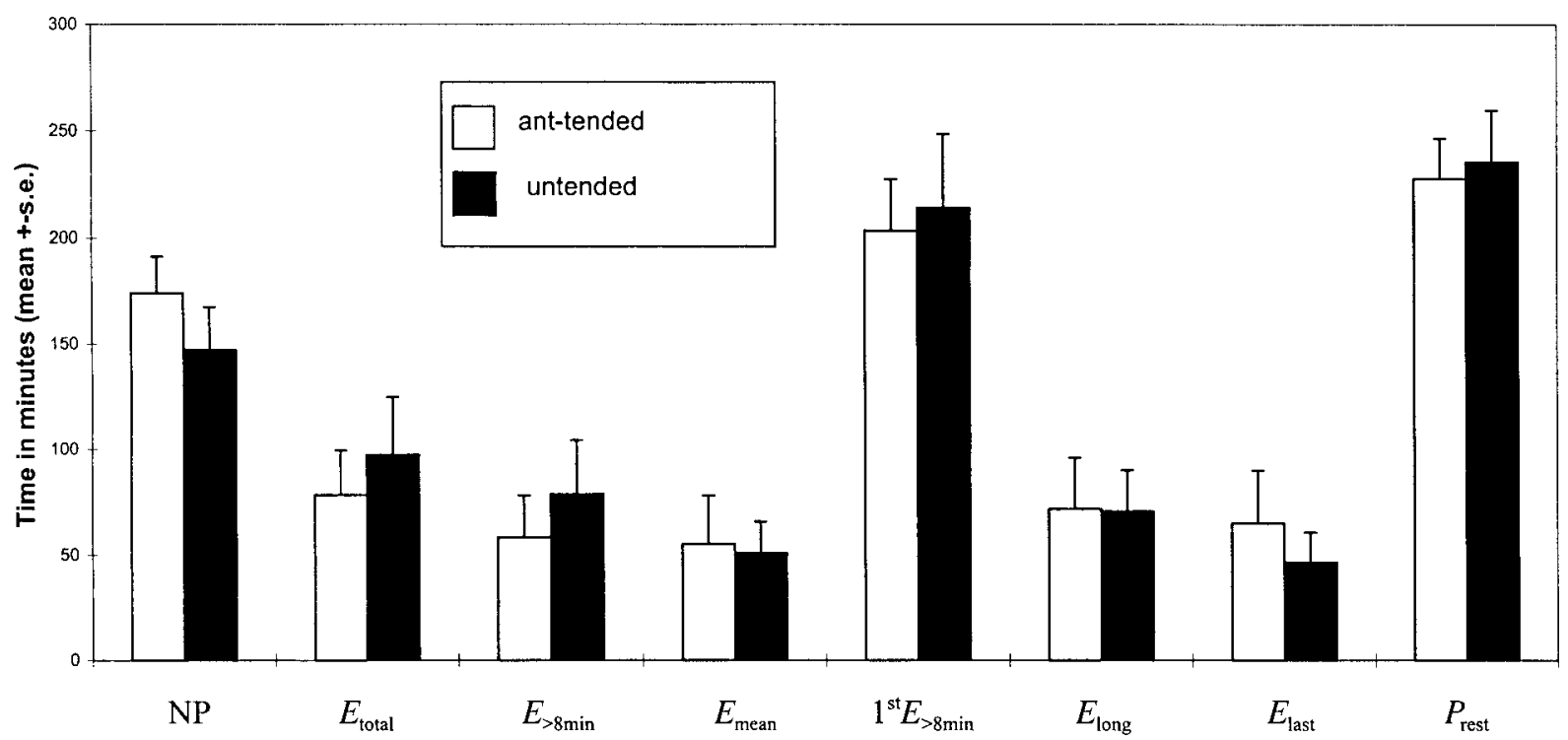

Behavioural Patterns

Figure 1. Durations of aphid behavioural parameters in the experiment (means and standard errors).

Differences between ant-tended and untended individuals were then analysed separately for the first and second $4 \mathrm{~h}$ periods. There were no significant differences in behaviour between ant-tended and untended aphids in either of the $4 \mathrm{~h}$ periods for any of the measured variables ( $t$-tests; $\mathrm{P}>0.05$; Figure 2 ).

A regression analysis indicated that the number of days without ant-attendance before the start of the experiment had no significant effect on any variable measured (linear regressions; $\mathrm{P}>0.05$ ).

\section{Discussion}

EPG-recording does not affect aphid-ant interactions. In this study, the electrical penetration graph (EPG) technique was employed for the first time to analyse the effects of ant tending on aphid behaviour. In the ant treatment, the wire could potentially have affected ant worker behaviour or interfered with the EPG signals. However, ants appeared to be unaffected by the presence of the wire. Workers readily tended aphids at high levels comparable to previous studies with single M. fuscoviride (Flatt \& Weisser, 2000) and no attacks on the wire were observed. When the ants touched the wire, EPG signals were still recognisable. Because we used two ant colonies, aphids from several different colonies, and a new plant for each replicate, the successful use of EPG was not due to choosing a particular aphid-ant-plant combination. We therefore conclude that the EPG technique is appropriate for measuring aphid behaviour during ant-aphid interactions.

Role of the experimental approach in explaining the lack of effects of ant-tending on aphid behaviour. The main result of our study is that EPG analysis did not reveal any differences between the behaviour of tended and untended aphids. In particular, anttended aphids did not spend more time penetrating sieve elements which may have reflected higher levels of nutrient intake. Even though the time spent in sieve elements is not directly linked with the actual amount of sap ingested, there is ample evidence for a strong relationship between the duration of behavioural parameters as measured by EPG and sap ingestion. For example, Lei et al. (1997) found a highly positive correlation between times of stylet penetration of the sieve elements and times of honeydew excretion by whiteflies. Spiller (1988) found that in resistant plants, the decrease in aphid honeydew production is associated with a decrease in the time spent ingesting from the sieve elements. Thus, our hypothesis that ant-tended individuals spent more time feeding in the sieve elements than untended aphids, which would have offered an explanation for the higher fitness of ant-tended individuals (Flatt \& Weisser, 2000), has to be rejected. Before discussing how ant tending could 


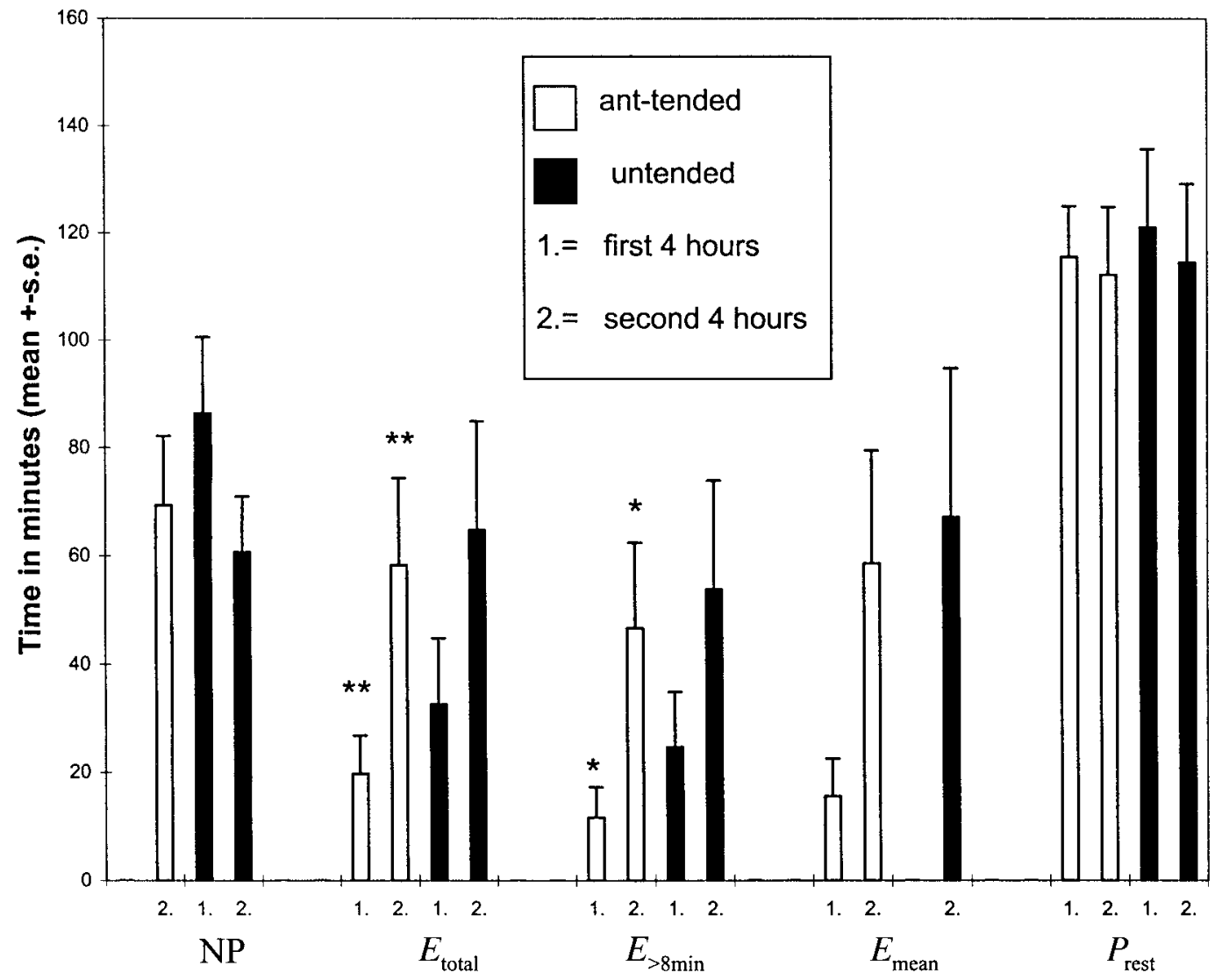

Behavioural Patterns

Figure 2. Durations of aphid behavioural parameters in the first and second half of the experiment (means and standard errors). Significant differences between the two time periods in the ant-tended treatment are marked with asterisks ( ${ }^{*}$ for $\mathrm{P}<0.05,{ }^{* *}$ for $\mathrm{P}<0.01$ ). The variables $\mathrm{NP}$ and $E_{\text {mean }}$ showed significant ant colony or aphid colony effects and were therefore not used for these analyses.

influence aphid behaviour in a way not detectable by EPG analysis, we comment on how the way the behaviour was analysed in our experiment could have affected the results.

In the analysis of the EPG waveforms, we were not able to distinguish between E1 (at the intracellular voltage level) and E2 within the $E_{\text {total }}$ waveform. This was probably due to the high level of noise within the $\mathrm{E}$ waveform. If ant tending selectively decreases $\mathrm{E} 1$ and increases E2 without changing the total time of sieve element penetration $(\mathrm{E} 1+\mathrm{E} 2)$, this change in the ingestion time period would not be detected by analysing only the total time of sieve element penetration $\left(E_{\text {total }}\right)$. However, the waveform of central interest in our study was total time of sieve element penetration. Thus, the distinction between E1 and E2 waveforms was not crucial for answering our main question. In addition, an $\mathrm{E}$ waveform that lasts longer than $8 \mathrm{~min}\left(E_{>8 \mathrm{~min}}\right)$ is generally regarded to represent sieve element acceptance and ingestion (Tjallingii, 1990). Because there were no significant differences in the $E_{>8 \min }$ parameter between ant-tended and untended individuals, any undetected changes in the proportions of E1 and E2 are restricted to the first $8 \mathrm{~min}$ of each penetration and are therefore unlikely to be a confounding factor.

Metopeurum fuscoviride can frequently be observed walking or dropping off the plant in its natural habitat (W. Völkl, W. W. Weisser, unpubl.). Walking or dropping off the plant is therefore not induced solely by the experimental set-up. In the experiments, dropping mainly occurred when aphids, after having walked for some distance, were held back by the wire. The time off the plant was minimized by putting fallen aphids immediately back on the plant. The interruption of feeding, caused by falling off the plant of an aphid, 
should therefore not be substantially different from the interruption of feeding due to walking.

In Flatt \& Weisser's (2000) experiment, individuals of $M$. fuscoviride were followed throughout their life-time and significant benefits of ant-attendance for aphid survival and fecundity were detected. In contrast, the measurement period of $8 \mathrm{~h}$ used in the present study represents only a small part of the life of an aphid. It is possible that longer periods of continuous observation are necessary to detect changes in aphid behaviour. Additionally, observational studies suggest that untended $M$. fuscoviride individuals have difficulties excreting honeydew (W. Völkl, unpubl.) which may be a proximal cause for their poor performance. Because the EPG-technique requires handling of individuals, it is possible that the physical contacts involved may have triggered honeydew release in these aphids, for example by the paint-brush used to put the fallen aphids back to the plants, even though in this study this was not noticed by the experimenter. Untended aphids that successfully excreted some honeydew may for a few hours be able to show a behaviour similar to tended aphids before the lack of ant workers becomes noticeable. Future studies should therefore aim at using longer time intervals to cover a larger part of the aphid life. Studies using immature instars may additionally provide further insight into the effects of ant tending on aphid feeding early in life. There are two reasons, however, that suggest that longer measurement periods may not yield different results. First, differences in behavioural parameters between anttended and untended individuals tended to be smaller rather than larger in the second four hour period than in the first 4-h period (Figure 2). Second, there was no clear indication that aphids needed time to get used to the EPG system (Figure 2).

In the present study, aphids were kept singly on plants whereas in the field $M$. fuscoviride is often found in large colonies. Individual aphids or small colonies however, are frequently observed in the field, for example when a single foundress colonises a plant (Flatt \& Weisser, 2000; W. W. Weisser, unpubl.). In Flatt \& Weisser's (2000) study, aphids were also reared singly on plants and a strong positive effect of ant tending was observed. Thus, low aphid density is unlikely to have been a confounding factor, in particular as ant tending levels were high.

In our experiments, temperature in the laboratory ranged from $24{ }^{\circ} \mathrm{C}$ to $29{ }^{\circ} \mathrm{C}$ which was rather high and might be suspected to have caused aphids to spend less time penetrating sieve elements. However, M. fus- coviride feeds on the upper part of the tansy shoot in sun-exposed habitats so that temperatures of $25^{\circ} \mathrm{C}-$ $30^{\circ} \mathrm{C}$ are not uncommon for this species in its natural habitats. Ant behaviour was not negatively affected by this temperature range as tending levels were similar to those observed in the experiments of Flatt \& Weisser (2000).

Finally, individuals of M. fuscoviride spent $16 \%$ and $20 \%$ of the $8 \mathrm{~h}$ period in the E waveform in the tended and untended treatment, respectively. Compared to some other studies, this appears to be a rather low percentage. For example, in $6 \mathrm{~h}$ recordings, the percentage of total time spent in $\mathrm{E}$ waveform was around $50 \%$ in A. pisum and for $8 \mathrm{~h}$ recordings about $77 \%$ (on suitable plants) and 38\% (on resistant plants) in S. avenae (Tjallingii, 1986; Caillaud et al., 1995). However, short periods of sieve element penetrations were, for example, also found in six hour recordings of Brevicoryne brassicae L., where individuals spent only about $25 \%$ of their time penetrating sieve elements (Tjallingii, 1986). Thus, the time spent in the sieve elements clearly depends on the biological system that is under study and the low percentage of total time in E waveform found in our study does not necessarily represent a problem of the experimental conditions.

Alternative mechanisms. There are at least two alternative behavioural mechanisms that could explain the increased fitness of ant-tended individuals and would be consistent with the results of our measurements. One possible mechanism is that ant tending increases the rate of sap uptake in aphids. Aphids would then benefit from ant-tending without any change in the time spent in the sieve elements. This mechanism would also result in an increased honeydew production as reported by several studies (Herzig, 1937; Banks \& Nixon, 1958; Buckley, 1987). A second possible mechanism is that ant-tending may increase the efficiency of nutrient uptake. In this case, increased fitness would not be associated with increased honeydew excretion, but the composition of the honeydew would be changed.

To test the first mechanism, it is necessary to measure honeydew production. Measurements of honeydew production of ant-tended aphids require a continuous observation of a single aphid to count the droplets of honeydew that are removed by the ants and a measurement of the volume of the honeydew droplet, as described by Herzig (1937). However, it may also be possible to quantify the amount of phloem sap in- 
gested through a careful analysis of the E waveform. A correlation may be found between the periodicity of the sinusoidal $\mathrm{E}$ wave and the amount of honeydew production, in absence of ants (Y. Rahbé, pers. commun.). If this correlation exists, it is then necessary to analyse the effect of ants on the $\mathrm{E}$ wave periodicity. If the ants increase the periodicity of the $\mathrm{E}$ wave, it is possible to conclude that the ants do increase the honeydew production and therefore increase the sap uptake. Also indirect measures such as radioactive labelling may provide some estimates of ingestion rates (Banks \& Nixon, 1958). To test the second mechanism, a comparison of the composition of the honeydew of tended and untended aphids would be necessary, as described by Völkl et al. (1999). A combination of EPG analysis with quantitative measurements of sap ingestion or honeydew composition analysis is likely to reveal further insights in the mechanisms underlying the interaction between ants and aphids.

To summarise, we suggest that the EPG technique is appropriate for the measurements of aphid behaviour in aphid-ant interactions. There were no significant differences in the duration of sieve element penetration or in any other analysed feeding-related EPG parameters between ant-tended and untended individuals. Before making any definitive conclusion there is, however, a need for complementary experiments that will allow the distinction between the EPG-waveform E1 (salivation only) and E2 (salivation and ingestion).

\section{Acknowledgements}

Support for this study came from grant no. 3100053852.98 of the Swiss National Foundation (WWW), the Josef und Olga Tomcsik Foundation (GR) and the Emilia Guggenheim-Schnurr Foundation (GR, TF). We thank Wolfgang Völkl, Yvan Rahbé, and two anonymous referees for constructive criticism of previous versions of this manuscript.

\section{References}

Banks, C. J., 1958. Effects of the ant, Lasius niger (L.) on the behaviour and reproduction of the black bean aphid, Aphis fabae Scop. Bulletin of Entomological Research 49: 701-714.

Banks, C. J. \& H. L. Nixon, 1958. Effects of the ant, Lasius niger L., on the feeding and excretion of the bean aphid, Aphis fabae Scop. Journal of Experimental Biology 35: 703-711.
Buckley, R. C., 1987. Interactions involving plants, Homoptera and ants. Annual Review of Ecology and Systematics 18: 111-135.

Caillaud, C. M., J. S. Pierre, B. Chaubet \& J. P. Di Pietro, 1995. Analysis of wheat resistance to the cereal aphid Sitobion avenae using electrical penetration graphs and flow charts combined with correspondence analysis. Entomologia Experimentalis et Applicata 75: 9-18.

Chongrattanameteekul, W., J. E. Foster \& J. E. Araya, 1991a. Biological interactions between the cereal aphids Rhopalosiphum padi (L.) and Sitobion avenae (F.) (Hom., Aphididae) on wheat. Journal of Applied Entomology 111: 249-253.

Chongrattanameteekul, W., J. E. Foster, R. H. Shukle \& J. E. Araya, 1991b. Feeding behavior of Rhopalosiphum padi (L.) and Sitobion avenae (F.) (Hom., Aphididae) on wheat as affected by conspecific and interspecific interactions. Journal of Applied Entomology 111: 361-364.

Febvay, G., Y. Rahbé \& M. van Helden, 1996. MacStylet, software to analyse electrical penetration graph data on the Macintosh. Entomologia Experimentalis et Applicata 80: 105-108.

Flatt, T. \& W. W. Weisser, 2000. The effects of mutualistic ants on aphid life-history traits. Ecology 81: 3522-3529.

Herzig, J., 1937. Ameisen und Blattläuse. Zeitschrift für Angewandte Entomologie 24: 367-435.

Lei, H. W. F. Tjallingii \& J. C. van Lenteren, 1997. Effect of tethering during EPG recorded probing by adults of the green-house whitefly. Journal of Applied Entomology 121: 211-217.

McLean, D. L. \& M. G. Kinsey, 1964. A technique for electronically recording aphid feeding and salivation. Nature 202: 1358-1359.

Nixon, G. E. J., 1951. The association of ants with aphids and coccids. Commonwealth Institute of Entomology, London, 36 pp.

Prado, E., 1997. Aphid-plant interactions at phloem level, a behavioural study. Thesis University of Wageningen, Wageningen, the Netherlands.

SAS Institute, 1989. SAS/STAT user's guide: Basics. Version 6.04: SAS Institute, Cary, North Carolina, USA.

Spiller, N. J., 1988. Electronic recording of plant penetration by the cereal aphids Rhopalosiphum padi and Metopolophium dirhodum on resistant and susceptible wheat seedlings. Annals of Applied Biology 112: 471-478.

Tjallingii, W. F., 1986. Wire effects on aphids during electrical recording of stylet penetration. Entomologia Experimentalis et Applicata 40: 89-98.

Tjallingii, W. F., 1990. Continuous recording of stylet penetration activities by aphids. In: R. K. Campbell \& R. D. Eikenbary, Aphid-Plant Genotype Interactions, Elsevier Science Publishers B.V., Amsterdam, pp. 89-99.

Tjallingii, W. F. \& A. Mayoral, 1992. Criteria for host acceptance by aphids. In: S. B. J. Menken, J. H. Visser \& P. Harrewijn (eds), Proceedings 8th International Symposium Insect-Plant Relationships. Kluwer Academic Publisher Dordrecht, the Netherlands, pp. 280-282.

Völkl, W., J. Woodring, M. Fischer, M. W. Lorenz \& K. H. Hoffmann, 1999. Ant-aphid mutualisms: the impact of honeydew production and honeydew sugar composition on ant preferences. Oecologia 118: 483-491. 